\title{
Fatma Umul
}

\section{Nach dem Juni-Aufstand \\ Die diskursiven Veränderungen der Frauenpolitik in der Türkei im Kontext der sozialen Widerstandsformen um den Gezi-Park'}

Der Juni-Aufstand ${ }^{2}$ in der Türkei 2013 kann als ein zivilgesellschaftlicher Widerstand verstanden werden, der die Konsequenzen einer neoliberalen Wirtschaftspolitik sichtbar machte. Als Reaktion auf die Repressionsmaßnahmen fanden sich Akteur_innen aus unterschiedlichen politischen, kulturellen und religiösen Kontexten und divergenten Interessen im sozialen Moment des Aufstands zusammen: Feministinnen, LGBTQ-Menschen, Linke, Anarchist_innen, Nationalist_innen, Kurd_innen, Alevit_innen Nicht-Bürger_innen und Bürger_innen, die zuvor wenig in politische Gruppierungen involviert waren. Bei der Thematisierung der Gezi-Proteste wird die heterogene Zusammensetzung der Demonstrant_innen als eines der charakteristischen Merkmale herausgestellt, neben der selbstorganisierten, nicht hierarchischen Gemeinschaft und den neuen Kommunikationsformen. So zählt etwa der Verein Taksim Solidarität 128 politische und gesellschaftliche Gruppierungen, die die Proteste unterstützten (Taksim Dayanışması 2014).Zahlreiche Protagonist_innen wurden unter diesem Dachverband aber nicht vertreten. Insbesondere waren Frauen bei den Treffen des Dachverbandes mit Politiker_innen der AKP, obwohl sie knapp über die Hälfte der Protestierenden ausmachten, nicht repräsentiert. ${ }^{3}$ Zeichnet die He-

1 Ich danke den Aktivistinnen der Frauenhausstiftung Mor Çatı, der PROKLA-Redaktion, Susanne Bauernfeind, Dorothee Engelhardt, Vincent Gengnagel, David Pithan, Katrin Rackerseder und Chris Wilpert für ihre Anmerkungen und die Bereitschaft zur Diskussion.

2 Die Bezeichnung Juni-Aufstand wird in diesem Aufsatz gleichbedeutend zu der Bezeichnung Gezi-Aufstand verwendet. Erstmaligwurde diese Bezeichnung von Errol Babacan im Artikel in der PROKLA 173 mit dem Titel: Der Juni-Aufstand in der Türkei, verwendet.

3 Für eine Studie wurde zwischen dem 6. und 8.Juni 2013 im Gezi-Park 4.411 Menschen zu Geschlecht, politischen Einstellungen, Bildungsgrad, Nutzung der sozialen Medien usw. befragt. Demzufolge beträgt der Anteil der Frauen im Park 50,8 Prozent und der Männer 49,2 Prozent. URL: http://www.konda.com.tr/tr/raporlar/KONDA_GeziRaporu2014. pdf, Zugriff: 4.1.2014. 
terogenität der Protestierenden eine Massenmobilisierung aus? Kann von einem Paradigmenwechsel gesprochen werden, der sich über die letzten Jahre hinweg schleichend aber sicher etablieren konnte? Wie findet ein Wechsel von einem Wertesystem zum anderen statt und wie kann dies sichtbar gemacht werden?

In diesem Aufsatz werde ich ausgehend von der Prämisse der Heterogenität innerhalb der Gezi-Park-Besetzer_innen, die Teilnahme der Frauen und die feministische Kritik an der AKP-Regierung näher untersuchen. Ziel ist es, eine differenzierte Analyse der Gezi-Proteste mit Blick auf die Hintergründe und diskursiven Entwicklungen bis zum Zeitpunkt des Ausbruchs der Proteste zu ermöglichen und somit zum besseren Verständnis beizutragen. Im ersten Teil werde ich herausarbeiten, wie das ideologische Konzept der Kernfamilie, als notwendige Voraussetzung für das Bestehen einer Nation, als ein mögliches Erklärungsmuster für die Entwicklungen der Frauenpolitik in der Türkei herangezogen werden kann. Daran anschließend werde ich die Veränderungen zwischen 2004 und 2014 in der Frauenpolitik nachzeichnen und anhand von Beispielen aus Interviews diese Kritik an der bestehenden Staatspolitik konkretisieren. Im zweiten Teil des Aufsatzes möchte ich die Widerstandsformen der Feministinnen innerhalb des besetzten Parks den diskursiven Entwicklungen in der Frauenpolitik entgegensetzen, um die Rekonstruktion der Gründe für den Ausbruch der Proteste im Juni 2013 zu ermöglichen.

\section{Die Verschränkung der Idee von der neuen Türkei mit dem Konzept der Kernfamilie}

Erdoğan hielt in der Nacht der Kommunalwahlen am 30. März 2014 seine vierte Balkonrede. Auffällig war, neben seinem überzogenen Auftritt mit seiner Familie, die rhetorische Anspielung auf die neue Türkei: „Meine Schwestern und Brüdern, ihr habt im Kampf um die Unabhängigkeit die neue Türkei verteidigt" (vgl. Umul 2014). Die Entstehung eines politisch autoritären Systems und die eingeleiteten wirtschaftspolitischen Veränderungen werden im Alltag von kulturell konservativen Praktiken begleitet. Dabei stellen die Veränderungen in der Frauenpolitik den ersten Schritt hin zu einem lang anhaltenden Paradigmenwechsel dar (vgl. Ataç 2009; Karakas 2013). Nükhet Sirman (2008) bietet einen Erklärungsansatz dafür, wie die Herstellung von vergeschlechtlichten Subjekten in der Türkei vonstattenging (ebd.: 315). In ihrer historischen Darstellung der türkischen Staatsgründung analysiert Sirman, wie die Staatsbürgerschaft als familiäre Staatsbürgerschaft diskursiv entstanden ist, um so den Paradigmenwechsel zur Nation zu begründen (ebd.: 297). So untermauert sie ihre These, dass die „Vorstellung von der Kernfamilie im gleichen Zug mit der Herstellung des Nationalstaates als 
modern stattfand“ (ebd.: 297). Das große Haus, d.h. das Zusammenleben nach alten Wertesystemen (vgl. ebd.: 302), findet in der Kernfamilie einen Ort der Reproduktion - in Form von Liebe zu einer Person und gleichzeitig zur Nation. In diesem Sinne konstituierte die gleichzeitige Etablierung der Kernfamilie und des türkischen Nationalstaates ein historisches Moment, die Familie, worin sich das Alte mit dem vermeintlich Neuen stärkte, um es als Selbstverständlichkeit zu reproduzieren. In einem immer weiter wachsenden Familien- und Gemeinschaftsgefüge würden die Mitglieder mit der Zeit einen Platz zugewiesen bekommen (vgl. Sirman 2008: 302). Dieser Platz wird mit Macht versehen und bestimmt parallel die Position, die das Individuum innerhalb des Staates einnimmt. Die Positionsbestimmungen nach der alten Ordnung werden in einem vermeintlich modernen Rahmen weiterhin selbst in Diskursen verfestigt. Die Position der Frau in der Familie wurde durch ihre Unterordnung definiert. Diese hierarchische Einordnung der Frau kennzeichnet für Sirman den Unterschied zum Westen, wo der geschlechtsneutrale Bürger unabhängig von seiner Rolle in der Familie konstituiert wurde. In der Türkei hingegen gab es die diskursive Transformation zur türkischen Staatsbürgerschaft, die über die Definition von Geschlechterrollen funktionierte und das Verständnis von der Kernfamilie etablierte. Die Liebe zur Nation gilt als Rechtfertigung, wenn die alte Ordnung kritisiert wird; gleichzeitig ermöglicht sie neue Hierarchien und legitimiert die Vorherrschaft der Männer, sowohl als Staatsbürger als auch als Ehemänner in der Familie. Die Unterordnung der Frau kann derart im Alltag, innerhalb der Familie und des Staates, weiterhin praktiziert werden (vgl. ebd.: 305f.).

Wenn Sirmans eine Kontinuität zwischen der historischen Konstituierung der Staatsbürgerschaft als Familisierung der Staatsbürgerschaft in der Türkei aufzeigt, dann wird deutlich, dass der aggressive Appell von Erdoğan an Frauen, drei bis fünf Kinder zu gebären oder die Verteilung von Hilfskrediten für junge Menschen, die unter 25 Jahren heiraten, kein Ausdruck einer neueren Entwicklung ist. Die Förderung der Kernfamilie und die damit zusammenhängende nationale Identität wurden in den letzten Jahren bei der kritischen Thematisierung der Entwicklungen in der Türkei im gleichen Atemzug mit wirtschaftlichen Entwicklungen genannt (vgl. Babacan 2014; Ataç 2009), jedoch nicht analysiert. Die Idee von der vermeintlichen Modernität der Kernfamilie, die historisch eine besondere Stellung hat, ermöglichte Erdoğan, eine neue Türkei zu etablieren, die sich auf ein politisch autoritäres System stützt, den Alltag in eine kulturell-konservative Richtung zu lenken und mit einer ökonomisch liberalen Wirtschaftspolitik den Schein eines modernen demokratischen Staates zu wahren. Karakaş stellt im Bezug auf die aktuellen Entwicklungen fest, dass der größte Erfolg der AKP nicht die Reformpolitik ist, sondern der eingeleitete Paradigmenwechsel. Der Islam, der unter Atatürk als rückschrittlich angesehen wurde, wird jetzt als Modernisie- 
rungsfaktor etabliert (Karakaş 2013: 25). Im Rahmen dieses Veränderungswillens der Machthabenden wird die Verteidigung der Kernfamilie propagiert, da diese ein höchst wichtiges Gut ist, das es zu kontrollieren und zu disziplinieren gilt.

\section{Die diskursiven Veränderungen in der Frauenpolitik der Türkei zwischen 2004 und 2014}

Um die Entwicklungen im Juni-Aufstand und die Kritik der Feministinnen an den politischen Entwicklungen der letzten Jahre nachvollziehbar zu machen, müssen die diskursiven Entwicklungen und Veränderungen davon analysiert werden, was als richtige Familie gilt. Im Folgenden wird anhand von exemplarischen Beispielen der Verlauf der familienpolitischen Interventionen der AKPRegierung dargestellt.

Im Jahre 2004 bekommt im Zuge der Diskussion um getrenntgeschlechtliche Studierendenwohnheime die Debatte über Ehebruch erhöhte Aufmerksamkeit. Nach der alten Gesetzgebung in den ersten Jahren der türkischen Republik (1926) wurde weiblicher und männlicher Ehebruch bestraft. Während die Definition für den „weiblichen“ Ehebruch jedoch bei einer sexuellen Beziehung mit einem Mann eintrat, galt als „männlicher“ Ehebruch, wenn der Mann mit einer anderen Frau, an einem anderen Ort zusammenlebte, etwa in einer gemeinsamen Wohnung. Im Jahre 1996 entschied das Verfassungsgericht das Gesetz, worin männlicher Ehebruch geregelt wurde, abzuschaffen, da es einer Ungleichbehandlung gleichkäme. Die Gesetzesregelung für „weiblicher Ehebruch “ wurde erst 1998 abgeschafft (vgl. Kadın Hukuku 2003). Die Debatte um Ehebruch, die im Jahre 2004 erneut auf der Tagesordnung stand, ist für die Wählerschaft der AKP-Partei von Bedeutung. Die AKP-Wählerschaft forderte um jeden Preis gesetzliche Regelungen, weil die Institution Familie aufrechterhalten bleiben sollte. Es sollte sanktioniert werden, was die familiäre Gemeinschaft strapaziert. Die Realisierung der Gleichstellung von Frauen und Männern trete dann ein, so Erdoğan, wenn auf der gesetzlichen Ebene weiblicher und männlicher Ehebruch gleich definiert werde und gleiche Konsequenzen für beide Geschlechter folgen. Die Verrechtlichung des Ehebruchs wird wie folgt begründet: „Familie ist bei uns eine heilige Institution. Solange die Institution Familie stark war, war auch diese Nation stark. Sobald die Institution Familie geschwächt wird, sind Länder zum Scheitern verurteilt“ (Zina Yasası 2004). ${ }^{4}$ Diese Aussage macht deutlich, dass die propagierte gesetzliche Gleichstellung die Stärkung der Familie zum Ziel hat und

4 Zitate aus den türkischen Medien und Interviewpassagen im folgenden Text wurden alle von der Autorin ins Deutsche übersetzt. 
nicht die Aufhebung der Diskriminierung von Frauen. Das Konzept der Familie dient der Stärkung der Nation und soll deshalb als ,heilig“ etablieren werden. Die Ablehnung der Familie bedeutet demnach „sich selbst zu leugnen“ bzw. die Türkei als Nation zu leugnen. Aufgrund des in- und ausländischen Protests konnte die Gesetzesregelung des Ehebruchs nicht durchgesetzt werden. Die AKP erntete darauf hin Kritik von Parteien, die auf dem politischen Spektrum noch weiter rechts liegen.

Im Jahre 2008 forderte Erdoğan drei bis fünf Kinder von jeder Frau, um die Familie und die Nation zu stärken. Diese Forderung wird mit dem Ziel des wirtschaftlichen Aufschwunges begründet:

Wenn wir eine starke Nation sein wollen, dann brauchen wir starke Familien. Starke Familien mit mindestens drei Kindern. [...] Es gibt ein Geheimnis in der Wirtschaft. Das Geheimnis ist der Mensch. Wenn es Menschen gibt, gibt es Kapital, Konsum, Produktion und Investitionen (3 Çocuk Talebi 2013)

An diesem Beispiel ist die Verschränkung der Nation mit dem Kapital zu beobachten. Damit wird eine klar definierte und abgesteckte Rolle der Frau zur Voraussetzung für das Selbstverständnis des konservativen Staates. Weitere gesetzliche Modifikationen, wie die des Bildungs- und des Krankenversicherungssystems, des Gewaltschutzgesetzes, die Flexibilisierung der Arbeitsverhältnisse und die Diskussion um die Etablierung der Krippen und Kindergartenplätze, impliziert eine klar definierte Rolle der Frau, auch wenn der Begriff Frau nicht vorkommt. Wie Sirman erläutert, fand zur türkischen Staatsgründung ein Paradigmenwechsel hinsichtlich der Rolle der Frau und hinsichtlich der Organisation des Zusammenlebens als Kernfamilie statt. Ähnliche diskursive Veränderungen sind in der AKP-Ära zu beobachten. So wird die Funktion der Frauen als Mütter der Nation weiter gefestigt.

2010 sagte Erdoğan erstmals: „Ich glaube nicht an die Gleichberechtigung zwischen Frauen und Männern. Deshalb bevorzuge ich die Bezeichnung Chancengleich heit. Frauen und Männer sind unterschiedlich, sie sind unzertrennlich “ (Eşit olamaz 2010). Diese Aussage lässt darauf schließen, dass die Annahme der Unzertrennlichkeit von Frauen und Männern - d.h. der Heterosexualität - im Kern homosexuelle Beziehungen als abnormal charakterisiert und dementsprechend zu einer radikalisierten Ablehnung von Homosexualität unter seiner Wählerschaft führt. Eine ähnliche, jedoch zugespitzte These vertrat er im November 2014 und bestätigt seine frühere Aussage: „Frauen und Männer kann man nicht gleichstellen, da sie von Natur aus anders sind“ (Fitrat Değil Anayasa 2014). Als Gegenreaktion wurde eine Erklärung von 59 Frauen- und LGBT-Organisationen veröffentlicht, in dem die Vorstellung einer natürlichen menschlichen Natur zurückgewiesen und stattdessen die Umsetzung der in der Verfassung geregelten Gleichstellung gefordert wurde. Da internationales Recht 
vor nationalem Recht gilt, wird in dem Schreiben die Verpflichtung der Türkei gegenüber den internationalen Abkommen hinsichtlich der Gleichstellung der Frauen und Männer gefordert (vgl. ebd.).

Ähnliche Meinungen und Thesen werden von AKP-Minister_innen vertreten. Bei der Diskussion um die Arbeitslosenquote wurde die Minderung der Anzahl der arbeitenden Frauen als eine Methode in Erwägung gezogen, um eine geringe Arbeitslosenquote zu erreichen. Demzufolge sollen Männer die von Frauen verrichteten Jobs übernehmen, da das Arbeitsangebot auf dem Markt schlecht sei. Die Arbeitslosenquote würde demzufolge sinken, da dann Frauen die unbezahlte Reproduktionsarbeit zu Hause verrichteten und mehr Stellen für Männer zur Verfügung stünden (vgl. Kadın işleri 2009). Diese These vertritt im Jahr 2009 der seit 2007 amtierende Finanzminister Mehmet Şimşek: „Speziell in Zeiten der Krise suchen Frauen nach Arbeit. Die Arbeitslosenquoten steigen, weil Frauen in der Krise arbeiten wollen“ (Kadın istihdamı 2009). Der amtierende Umweltund Waldminister, Veysel Eroğlu, vertritt im gleichen Jahr dieselbe These und antwortet einer Frau, die nach einem Arbeitsplatz fragt, mit der Gegenfrage: „Reicht die Hausarbeit nicht?“" (Ev işi 2009).

Bei der Sitzung, des Hohen Rats der Richter und Staatsanwälte (HSYK) im Jahre 2011 wurde vorgeschlagen, in Fällen von Gewalt gegen Frauen, diese nicht als öffentliche Prozesse, sondern als Zivilrechtsprozesse zu behandeln, um die Verfahren zu beschleunigen und die Anzahl der Verfahren zu senken. Dabei sollten „Versöhnungskommissionen“ helfen. Wenn Mädchen unter 15 Jahren einwilligen, Geschlechtsverkehr zu haben, so der Vorschlag, dann sollte die Strafe gemindert werden. Frauen, die vergewaltigt wurden, sollten auf ein psychologisches Gutachten verzichten, um die Verfahren nicht unnötig zu verlängern. Ein ärztliches Gutachten sollte ausreichen. Bei der Verheiratung von minderjährigen Mädchen sollte bei Zustimmung des Mädchens auf eine Strafe für den Mann verzichtet werden. Frauen, die vergewaltigt werden, sollten ihre Vergewaltiger heiraten, um die die Verfahren zu beschleunigen und die Anzahl zu mindern (vgl. Kadın Evlensin 2011). Der Hohe Rat bestritt, den Katalog an Vorschlägen je aufgestellt zu haben. Diese Vorschläge habe es nie gegeben, so der HSYK bei einer Presseerklärung. In der Presseerklärung wurden zudem Journalist_innen aufgefordert, in Zukunft besser zu recherchieren, um das Vertrauen, das dem HSYK in der Öffentlichkeit entgegengebracht wird, nicht zu brechen (vgl. HSYK 2011).

Eine wichtige Zäsur in der Frauenpolitik war 2011, die erneut mit der Gleichstellungsthese und der Kernfamilie begründet wurde. Hierzu zählt die Restrukturierung des Kabinetts (vgl. Bakanlar Kurulu 2011), worin in einzelnen Ämtern organisierte Ministerien für Frauen, Kinder sowie behinderte und ältere Menschen unter dem Ministerium für Familie und soziale Politik zusammengefasst 
wurden. Diese Begriffsveränderung wird von Frauenorganisationen wie Mor Çatıals als Wendepunkt in der Frauenpolitik verstanden. ${ }^{5}$

Die Verwendung des Begriffs Familie statt Frau, so wird kritisiert, sei Ausdruck einer Verdrängung der Frau vom Öffentlichen ins Private (Kadın Örgütleri 2011). Bei dieser Kritik steht die neoliberale Verwertung der unbezahlten oder schlecht bezahlten Reproduktionsarbeit, die als eine natürliche Aufgabe an Frauen delegiert wird, im Vordergrund. Diese begriffliche Veränderung tabuisiert zudem Gewalt gegen Frauen und führt sowohl auf der institutionellen als auch auf der gesellschaftlichen Ebene zur Benachteiligung der Frauen. Während die institutionelle Benachteiligung durch die Freisprechung von Vergewaltigern sichtbar wird, zeigt sich die gesellschaftliche Akzeptanz der Gewalt gegen Frauen etwa in der Zeitungsberichterstattung über Frauenmorde. ${ }^{6}$ Auffällig, so Mor Çatı , sind die steigenden Zahlen der Frauenmorde in den letzten Jahren - jeden Tag werden drei Frauen getötet, weil sie Frauen sind.

Hinzu kommen die Veränderungen des Bildungssystems mit der Kennzeichnung „4+4+4“ im Jahre 2012. Die Reformen in der Bildungslandschaft der Türkei wurden im Schuljahr 2011/2012 eingeführt. Zuvor wurde im Jahre 1998 die Schulpflicht für 8 Jahre eingeführt. Vor 1998 lag die Zahl der Mädchen, die eine Grundschule besuchten, bei 78 Prozent und 34 Prozent besuchten eine Mittelschule. Im Jahre 2012 besuchten 98 Prozent der Mädchen eine Grundschule und 62 Prozent eine Mittelschule. Im Zuge der „Reformen“ sollte die Schulpflicht für 8 Jahre beendet und ein neues System eingeführt werden, das in drei Blöcke von jeweils vier Jahren eingeteilt wird. Im ersten Block sind Schüler_innen von 5 bis 8 Jahren, im zweiten Block Schüler_innen von 9 bis 12 Jahren und im dritten Block Schüler_innen von 13 bis 16 Jahren. Die 8-jährige Schulpflicht wurde halbiert und damit die Möglichkeit wieder eingeführt, dass Schüler_innen nach dem zweiten Block entweder in Imam-Hatip-Schulen (religiöse Berufsschulen) oder in Berufsschulen gehen. Die erste Ausarbeitung der Bildungsreform beinhaltete die Option, bereits nach dem ersten Block im Alter von 8 Jahren das staatliche Bildungssystem zu verlassen. Während dies aufgrund der Kritik von Frauenorganisationen auf den zweiten Block verschoben wurde, kann der dritte Block von zu Hause aus, in Form

5 Die Frauenhausstiftung Mor Çatı wurde im Jahre 1990 in Istanbul gegründet. Neben einem Solidaritätszentrum besitzt sie ein autonomes Frauenhaus, das nach feministischen Prinzipien arbeitet. Neben psychologischer und juristischer Unterstützung für Frauen und Kinder dokumentiert sie die Entwicklung in der Frauenpolitik in der Türkei (vgl. ak 585).

6 So hieß die Überschrift eines Berichts in der Tageszeitung Takvim (30.6.2013) „Nakavt“ (Knockout), in dem der Streit zwischen einem Pärchen auf der Straße thematisiert wird. „Der Mann hat die Frau mit den Fäusten geschlagen“ heißt es. Auf dem Foto des Berichts liegt die Frau mit blutüberströmtem Gesicht auf dem Boden." (vgl. ak 585). 
von Fernschulen absolviert werden. Die geplante Einschränkung der Schulpflicht auf vier Jahre und die Option, bereits nach dem Ende der vierten Klasse eine religiöse weiterführende Schule zu besuchen, hat vielen Eltern die Möglichkeit eröffnet, Mädchen den Zugang zu regulären Bildungsabschlüssen zu entziehen. Ab dem zweiten Block, d.h. mit 12 Jahren, die staatlichen Schulen verlassen zu können, um von zu Hause einen weiterführenden Bildungsabschluss zu erlangen, birgt die Gefahr, dass Mädchen, statt in die Schule zu gehen, bereits früh verheiratet werden (vgl. Tolunay 2014). Die Bildungsreformen sind neben den strukturellen Änderungen auf der gesetzlichen Ebene, durch Eröffnung und Schließung von Schulen gekennzeichnet. Das Ratsmitglied der AKP in Çorum, Erhan Ekmekçi, kommentiert die hohe Zahl an Schülerinnen mit der folgenden Aussage: „Wenn Mädchen die Schule besuchen, finden Männer keine Frauen zum Heiraten“" (Eğitim 2012). Ebenfalls eine große Debatte riefen die angestrebten Regulierungen des Abtreibungsgesetzes hervor. Frauen werden im Verlauf dieser Debatte kriminalisiert. Wie bei allen Themen bezieht Erdoğan auch hier klar Stellung:

Ich bin ein Ministerpräsident, der eine geplante Geburt wie bei einem Kaiserschnitt ablehnt. Ich weiß, dass dies Schritte sind, die das Steigen der Bevölkerungszahl verhindern. [...] Ich sehe Abtreibung als Mord. Ja Abtreibung sehe ich als Mord - diese Aussage von mir wird in manchen Kreisen angeprangert - ich appelliere auch an die Mitglieder der Medien. Sie schlafen miteinander, stehen auf und schreien Uludere. ${ }^{7}$ Ich sage, jede Abtreibung ist ein Uludere (zit. nach Kürtaj Uluderedir 2012).

Die Forderung der Frauenorganisationen mit dem Slogan „Mein Bauch gehört mir" wird durch Aussagen wie diese delegitimiert. Zustimmung bekommt Erdoğan auch bei dieser Debatte von AKP-Abgeordneten. Melih Gökçek, der Oberbürgermeister von Ankara, vertritt seine These wie folgt: „Abtreibung ist Mord, keine darf sagen, das ist mein Körper, ich kann machen, was ich möchte. [...] Den Fehler der Frau muss das Kind tragen. Soll sie sich doch selbst umbringen" (Kendini öldürsün 2012). Der Gesundheitsminister von 2002 bis Anfang 2013, Recep Akdağ, schlägt bei einer Schwangerschaft aufgrund einer Vergewaltigung Folgendes vor: „Die Frau soll gebären, wenn es notwendig ist, wird sich der Staat um das Kind kümmern“ (Tecavüz Bebeği 2012). Weiterhin argumentiert die ehemalige Familienministerin Fatma Şahin mit dem Argument: „Das Recht auf Leben beginnt bereits im Mutterleib. Außerdem ist Abtreibung kein Verfahren der Familienplanung" (Kürtaj açıklaması 2012).

7 Uludere ist eine kleine kurdische Stadt nahe Şırnak. Am 28.12.2011 wurden dort Menschen mit F-16-Kampfflugzeugen angegriffen. 35 Menschen starben. 20 Menschen waren sehr junge Schulgänger_innen und transportierten Tabak und Benzin über die irakische Grenze. Es gab Proteste gegen diesen Anschlag, da das Passieren der Grenze bis zu diesem Zeitpunkt von den staatlichen Behörden meist toleriert wurde. Warum an diesem Tag Menschen angegriffen wurden, ist bis heute ungeklärt. 
Seit 1983 ist die straffreie Abtreibung bis zu zehn Wochen nach Empfängnis gesetzlich verankert. Zu der damaligen Zeit wurde die Abtreibung eingeführt, um die Bevölkerungszahl zu senken (Günel 2013: 26). Geburtenregulierung, gegen das Selbstbestimmungsrecht der Frauen, ist ein gängiges Instrumentarium, um Macht zu demonstrieren. Die derzeitige türkische Regierung wollte die Abtreibung nach der vierten Schwangerschaftswoche verbieten. Diese angestrebte Veränderung konnte aufgrund der verstärkten Kritik seitens der Frauenorganisationen nicht verwirklicht werden. Dafür wurden Einschränkungen in Bezug auf die Art der Geburt vorgenommen. Demzufolge ist ein Kaiserschnitt nur erlaubt, wenn entweder die Mutter oder das Kind lebensbedrohlich gefährdet ist. Der Eingriff in die Art der Geburt findet, wie im obigen Zitat aufgezeigt, ihre Begründung in dem Argument einer natürlichen Geburt. Die gesetzlichen Einschränkungen im Abtreibungsgesetz konnten nicht verändert werden. Es existieren jedoch informelle Strukturen: Die automatische Benachrichtigung der Eltern oder des Mannes im Falle einer Abtreibung, oder das Einschalten von Ärzt_innen und Krankenhäusern, die die Durchführung einer Abtreibung verweigern, sind Verfahren, die Frauen eine selbstbestimmte Entscheidung nicht gewähren.

Im Jahre 2012 organisierte das Familienministerium unter dem Motto: „Frauen als Unternehmerinnen "Weiterbildungsseminare für Frauen. Diese Kurse zielen darauf ab, dass Frauen die Männer beim Verdienst unterstützen, etwa durch Stricken und Nähen. Sie sollten die Fähigkeit erlangen, im Falle eines geringen Verdienstes des Familienvaters das Einkommen zu sichern, indem sie lernt, ihre Fähigkeiten zu vermarkten. Die wertschaffende Produktionsarbeit wird von der Reproduktionsarbeit getrennt. Dabei wird auf die unbezahlte Reproduktionsarbeit hingewiesen. Mit dem nicht wertschaffenden Charakter der Hausarbeit fällt auch eine angemessene Rentenversicherung weg, was eine finanzielle Abhängigkeit der Frauen von den Männern nach sich zieht. Diese Asymmetrie in der Beziehung macht sich beispielsweise beim Scheidungswunsch bemerkbar. Da im Falle einer Scheidung die finanziellen Hürden und die bürokratischen Rechtswege schwer zu bewältigen sind, bleibt die Verwirklichung einer Scheidung und damit ein selbstbestimmtes Leben für viele Frauen utopisch. Der Arbeitsmarkt bietet keine Stellen für Frauen aus sozial schwachen Teilen der Bevölkerung, da ihnen die Bildungswege durch die Reformen versperrt werden und zudem schlecht bezahlte Arbeitsplätze zunächst von Männern besetzt werden sollten.

Nicht zuletzt wurde die Funktion der Familie für die Nation nach den Protesten weiter gestärkt, indem beispielsweise Kredite für junge Menschen vergeben wurden, die unter 25 Jahren heiraten. Weiterhin werden, wenn junge Menschen bereits in der Schul- oder Universitätszeit heiraten, die Kredite erlassen und so die Gründung der Kernfamilie leicht gemacht (Faizsiz Çeyiz 2013). Das Sankti- 
onieren von gemischtgeschlechtlichen Wohngemeinschaften als eine nicht wünschenswerte Art des Zusammenlebens fällt ebenfalls in die Nachprotestphase.

Um die Position der Frauen innerhalb des türkischen Staatsapparates und der Gesellschaft näher zu untersuchen, habe ich in den bisherigen Ausführungen zunächst den Versuch unternommen, patriarchale Denkmuster aufzuzeigen, die dem Geschlechterverhältnis in der Türkei zugrunde liegen. Anschließend habe ich den Paradigmenwechsel in der Frauenpolitik dargestellt, um dadurch den Zusammenhang zwischen der Nation und dem Kernfamilienkonzept als einen diskursiven Ort, worin Machtstrukturen reproduziert werden, hervorzuheben. Im folgenden Teil werde ich die Widerstandsformen der Feministinnen innerhalb des besetzten Parks den diskursiven Entwicklungen in der Frauenpolitik entgegensetzen, um die Rekonstruktion der Gründe für den Ausbruch der Proteste im Juni 2013 zu ermöglichen.

\section{Soziale Widerstandsformen der feministischen Aktivistinnen}

Welche Rolle spielte die feministische Kritik innerhalb der Gezi-Kommune? Mit welchen Aktionsformen konnte sie sich im Kommunen-Diskurs etablieren? Anhand exemplarischer Aussagen werde ich zeigen, welche Aspekte die Protagonistinnen an der Frauenpolitik der AKP kritisieren. ${ }^{8}$ Gleichzeitig wird die Beschreibung der Ereignisse im Park als eine neue Form der Interaktion und Kommunikation dargestellt, in der sich neue Aktions- und Widerstandsformen etablieren konnten. Die Aussagen der interviewten Personen enthalten zwei Ebenen. Beim Vergleich der beiden Aussageebenen ist zwischen einer nicht-interaktiven Kritik und einer interaktiven Kritik zu unterscheiden. Nicht-interaktiv ist die Praxis der Frauenbewegung vor den Protesten. Dies führt Al-Rebholz auf das Verständnis des Verhältnisses zwischen Staat und Zivilgesellschaft zurück. Ein antagonistisches Verständnis würde weder dazu führen, dass ein neues Modell für das Verhältnis zwischen dem Staat und der Zivilgesellschaft etabliert wird, noch würde es Relevanz für eine feministische Politik haben, deren Hauptinteresse die Demokratisierungsprozesse hinsichtlich Geschlecht und anderer sozialer Verhältnisse sind (vgl. Al-Rebholz 2007: 222). Der Gehalt, der als neu bezeichneten interaktiven Kritik kennzeichnet an dieser Stelle den von Al-Rebholz rezipierten Begriff von Frasers Gegenöffentlichkeit. Hier wird Öffentlichkeit nicht nur als

8 Hierfür habe ich sieben Interviews mit Frauen geführt (Frau A bis G), die innerhalb der türkischen Frauenbewegung aktiv sind und sich als Feministinnen bezeichnen. In den offenen Interviews war meine Hauptfragestellung: „Wie hast Du dich im Gezi-Park als Frau ausgedrückt - mit welchen Protestformen?"Die Interviews wurden im März 2014 durchgeführt. 
diskursiver Schauplatz verstanden, sondern als Körperlichkeit: rebellische und kreative Formen des Sprechens, Verhaltensweisen, Selbstbehauptungen, Kleidungscodes, Raumeroberungen, neue Jargons, ein eigener Wortschatz gegen die herrschende offizielle Ideologie (vgl. Al-Rebholz 2011: 56). Auf der Grundlage dieser Unterscheidung werden im Folgenden die Interviews analysiert.

Auf der Ebene der nicht-interaktiven Kritik wird staatliche Politik abgelehnt. Diese Kritik wird durch die gängige Protestform Demonstrationen ausgedrückt. Polizeigewalt und die Verweigerung von transparenten und öffentlichen Verhandlungen seitens der Machthabenden verhindert, dass neue Protestformen sichtbar werden und damit die Möglichkeit der kollektiv-körperlichen Erfahrung im Alltag. Die repressive Unterbindung von alltagsrelevanten alternativen Handlungsstrategien bereitet den Weg für ein antagonistisches Verhältnis zwischen der Staatsmacht und den kritischen Kräften. Statt kommunikativer Verhandlungsstrategien und Aktionsformen werden Strategien der Ablehnung und Konfrontation erlernt und angewandt.

Die interaktive Kritik, wie sie im Gezi-Park praktiziert wurde, um „das Äußere“, den Staat, abzuwehren, ließ Verhandlungen zu und ermöglichte die Erweiterung der praktischen Protestformen. Unter anderem deshalb konnte die anfängliche Idee, einen öffentlichen Park für sich zu beanspruchen, zu einem öffentlich-politischen Raum werden, in dem sich differierende Standpunkte Gehör verschaffen konnten. Die kollektive Erfahrung, dass die Politik des Staates eine korrupte Wirklichkeit konstruiert, wie etwa die Unterlassung der Berichterstattung über die Proteste oder die mediale Darstellung der Protestierenden als Verräter_innen der Nation, führte zu der oft rezipierten Veränderung des Bewusstseins - und verlieh ihr eine Körperlichkeit (vgl. ebd. 2011: 56).

Vier von sieben Frauen treffen Aussagen über beide Ebenen, eine Frau thematisiert explizit die Veränderungen in der Frauenpolitik und zwei Frauen treffen Aussagen in Bezug auf die Aktionsformen der Feministinnen im Park. Die Veränderungen in der Frauenpolitik werden meist als Eingriff in das eigene Leben, als frauenfeindlich, sexistisch und heterosexistisch bezeichnet. Frau A drückt sich wie folgt aus:

Die Proteste waren generell Proteste gegen die AKP-Regierung. Die ist so frauenfeindlich, in jeder Form sexistisch, heterosexistisch. Dass es tausende Gründe gibt weshalb ich dort, mit meiner Frauenidentität und Frauenzuschreibung auch gekämpft habe.

Diese Kritik wird von Frau B, C und D ebenfalls geteilt. Wie bei Frau C werden die Veränderungen in der Familienpolitik als „Tropfen, der das Fass zum Überlaufen brachte" bezeichnet, der die persönliche Teilnahme an den Protesten begründete. Das Argument „Ich war besorgt darüber, dass ein Eingriff in meine Lebenswelt stattfand "diente in den meisten Publikationen der feministischen Zeitschriften (Feminist Politika, Amargi) in der Nachprotestphase als Begründung. Frau E 
geht konkret auf diese Entwicklung ein und kommentiert die hohe Teilnahme der Frauen an den Protesten wie folgt:

Mit den Gezi-Protesten konnten wir gegen eine frauenfeindliche Regierung unsere Stimme erheben. Über die Hälfte der Besetzenden im Park waren Frauen. Die frauenfeindliche Politik der AKP-Regierung spielt dabei eine große Rolle. Angefangen bei der Anzahl der Kinder, ob ich abtreiben darf oder nicht, die Berichterstattung der Gerichtsmediziner bei einer Vergewaltigung, dass mir ständig eine männlich dominierte Justiz gegenüber steht, dass die Gewaltschutz-Regelungen nicht praktisch umgesetzt werden, dass Frauen ständig umgebracht werden. In Gezi konnte ich meine jahrelange Rebellion ausdrücken. Ich habe gegen die männliche Herrschaft protestiert.

Als einzige unter den interviewten Frauen erwähnt sie die neuen Aktionsformen der Feministinnen im Park nicht. Dies kann repräsentativ in die Ebene der antistate-ideology eingeordnet werden. Frau D thematisiert die Illusion der Gleichstellung im Park, indem sie auf die fehlende Repräsentation der Frauen hinweist:

Gezi war nicht, wie es in den Medien gezeigt wurde, so sehr eine Frauensache. Ich habe mich vom Verein Taksim Solidarität (TD) nicht repräsentiert gefühlt. Nach den Protesten habe ich ein paar Mal an den Sitzungen der TD teilgenommen. Ich habe mich nicht als Teil und wahrgenommen gefühlt.

Hier zeigt sich ein Widerspruch zu der im Park erfahrenen Realität der Gleichstellung, trotz der Heterogenität und den damit einhergehenden Unterschieden in Bezug auf gender, race and class.

„Nicht mindestens drei Kinder, sondern mindestens drei Parks“ - unter diesem Slogan organisierten Feministinnen kurz nach der Räumung des Parks die „Stehende-Frau-Performance“.9 Der Übergang von der Straßen- und Barrikadenphase zur Besetzungsphase wird als der soziale Moment der Proteste wahrgenommen. Während der 15-tägigen Besetzung transformierte sich der Park von Tag zu Tag, sodass innerhalb weniger Tage eine selbstorganisierte Infrastruktur etabliert werden konnte. Bereits einen Tag vor Beginn der drei Tage andauernden Straßenkämpfe wies eine Rednerin ausdrücklich darauf hin, dass im Park kein Platz für Sexismus, Homophobie, Transphobie und sexuelle Belästigungen sei. Frau A erinnert sich wie folgt an die Präsenz der Feministinnen und der LGBT-I Aktivist_innen im Park:

In Gezi war eine offene Kommunikation, jeder hat versucht, auf den anderen einzugehen. Es war eine noch nie in meinem Leben erlebte Form von Solidarität. In der Mitte gab es einen Stand der LGBT-I Bewegung, dann gab es den feministischen Stand. Es

9 In Anlehnung und gleichzeitig in Abgrenzung zum sogenannten stehenden Mann, der auf dem Istanbuler Taksim-Platz die Polizeibrutalität still anklagte und hundert Nachahmer fand. Das einfache und stille Herumstehen an den unterschiedlichsten und ungewöhnlichsten Orten, machte es der Polizei schwer, einzugreifen, obwohl allen klar war, dass es eine Form des politischen Protests war. 
[Geschlechtsidentität] war die ganze Zeit ein Thema, weil es darum ging eine Utopie im Park - komplett unabhängig von der Gesellschaft außerhalb, einen neuen Raum zu schaffen. Deshalb war die ganze Zeit eigentlich neudefinieren und aushandeln ein Thema.

Mit der Präsenz eines feministischen Ortes, wie es Frau A schildert, beginnt die Besetzungsphase im Park. An dieser Schilderung ist zu erkennen, dass die Auseinandersetzung um Identität ein wichtiges Thema war. Die Form der Solidarität, die sie beschreibt, lässt darauf schließen, dass im Vordergrund stand, ständig Kommunikationsmöglichkeiten neu zu definieren und auszuhandeln, die Sexismus ausschließen. Einige Slogans spiegeln den doppelten Charakter des feministischen Widerstandes wider. Der Protestspruch: „AKP nimm deine Hände von meinem Körper" bezieht sich auf die Abtreibungsdebatte, „Nicht mindestens drei Kinder, sondern mindestens drei Parks“ verweist auf die 3-5-Kind-Forderung von Erdoğan. Der Slogan „Leiste Widerstand - nicht mit Sexismus, sondern mit Hartnäckigkeit“ hingegen thematisiert die sexistischen Slogans im Protestalltag. Der Slogan „Erdoğan, willst Du wirklich drei Kinder von mir?" enthält einen Widerstandsdiskurs sowohl gegenüber der Geburtenpolitik der Regierung als auch die Fragestellung, ob wirklich drei Kinder von einem "Çapulcu“ (Plünderer_in) erwünscht seien. Mit diesem Slogan wird auf der einen Seite die Unterdrückung als Frau und auf der anderen Seite als Protestierende ironisiert. Ein weiterer Slogan wie „Wir brauchen Freiräume ohne Tayyip und ohne sexuelle Belästigung “ lässt auf zwei Realitäten schließen. Freiräume ohne den Premierminister bzw. einen Mann verweist hier auf die Kritik an sexuellen Belästigungen in der türkischen Gesellschaft, sei es innerhalb des besetzten Gebietes oder außerhalb. Dass die Parkrealität nicht ein Ort ohne Sexismus war, beschreibt Frau B wie folgt:

Die Behauptung, es sei ein Ort ohne Sexismus gewesen, würde ich nicht vertreten. In der Tat war Gezi ein männlich dominierter Protest. Aber wenn man Gezi ohne zu romantisieren betrachtet, dann war es ein Ort, der Raum für Aushandlungen zugelassen hat. Bei diesen ganzen Vergleichen kann man dies generell als einen positiven Aspekt der Besetzungszeit in Betracht ziehen. Es gab auch Männer und Fußballfans, die sexistische Sprüche beim Vorbeigehen am feministischen Zelt benutzt haben. Aber man kann sagen, dass man normalerweise nicht so laut dagegen vorgegangen wäre, wenn es nicht den Zusammenschluss und das Selbstvertrauen wie wir sie im Park hatten, gegeben hätte. Normalerweise ging es mir nur bei Frauendemonstrationen so, dass ich mich sicher und organisiert gefühlt habe.

Frau C, D und G thematisieren die sexistischen Slogans im Park ebenfalls. Frau C schildert ihre erste Konfrontation mit der sexistischen Sprache des Widerstandes wie folgt:

Nachdem der Park besetzt war und ich zu Taksim gekommen bin, wurde ich mit sexistischen Sprüchen auf den Wänden konfrontiert. Geschimpft wurde über Erdogans Frau, Tochter und Mutter. Deshalb musste ich erst mal überlegen, auf wessen Seite ich war. 
Als Reaktion darauf wurde ein Protest organisiert, mit dem Ziel, die Protestsprache zu verändern. Hier wurden die Slogans mit der Farbe Lila übermalt, um die Protestierenden darauf aufmerksam zu machen. Diese Kritik wurde im Park mit Wohlwollen aufgenommen. Daraufhin thematisierte der anarchistische Fußballverein Çarsı die sexistische Sprache im Park. Schließlich wurde ein Workshop organisiert, um gemeinsam alternative Sprachfloskeln für den Widerstand zu finden. Die Veränderung der Protestsprache schildert Frau A mit dem folgenden Beispiel:

Ein Beispiel waren die öffentlichen Toiletten. Es gab einen Teegarten, der seine Toilette zur Verfügung gestellt hat. Da hing auf der einen Tür „Bay“ und auf der anderen Seite „Bayan“ d.h. die Seite von dem Herren. Das habe ich kritisiert und wir haben es dann umgeschrieben. Alle, sowohl Personen, die sich als Mann oder als Frau beschrieben haben, machten bei der Diskussion interessiert mit. Das war echt super, weil normalerweise, wenn man Sprichwörter als sexistisch ablehnt, wird man mit Abweisung konfrontiert.

Weiterhin wird von Frau B und Frau F trotz der existierenden sexuellen Belästigungspraxen das Gefühl der Sicherheit als Frau auf der Straße bemerkt. Frau B thematisiert dies wie folgt:

Es war ein ganzes Besetzungsgebiet, nicht nur der Gezi-Park. In diesem Zeitraum [Besetzungszeit vom 1. Juni - 15. Juni] fühlte ich mich so sicher wie noch nie auf den Straßen. Auch in der Nacht um drei sind die Straßen für uns [Frauen] zu einem sicheren Platz geworden. Straßen, die ich noch nie zuvor betreten hatte, waren in der Nacht sicherer als am Tag. Das, was im Park passiert ist, wirkte sich nicht nur auf Taksim und Umgebung, sondern auf die ganze Stadt aus.

Die Sicherheit auf der Straße aufgrund der Geschäftigkeit bleibt auch nach der Räumung des Parks ein Thema. Das Entstehen zahlreicher Diskussionsforen in Istanbul hat nach den Protesten Raum für Auseinandersetzungen gewährleistet. In feministischen Foren wurden Themen wie „Frau und Stadtentwicklung“ oder „sexuelle Belästigungen im Park“ diskutiert. Darauf verweisen Frau D und G. Ein interessanter Aspekt, der von Frau C und D geschildert wird, ist die nicht-hierarchische Organisation im Park. Dies und die kollektiven Entscheidungsmechanismen werden als explizit feministische Methoden hervorgehoben. Frau C erklärt:

Als Frauen beim Widerstand hatten wir uns so oder so Widerstandsformen im Laufe der Zeit angeeignet, wie die nicht-hierarchischen, kollektiven Entscheidungsmechanismen. Allein als Frau in einer männlich dominierten Gesellschaft auf die Welt zu kommen, fordert Widerstand. Zu sehen, dass sowohl in Gezi als auch danach, diese Widerstandsformen jetzt nicht nur innerhalb der Frauenbewegung Anwendung finden, sondern von gemischten Gruppen auch gewürdigt werden - lässt mich wissen, dass ich aufdem richtigen Weg bin.

Frau D fügt hinzu: „Deshalb habe ich mich als Frau sehr gut gefühlt im Park.“ Dass die Merkmale nicht-hierarchische Strukturen und kollektive Entscheidungsmechanismen laut den obigen Aussagen gängige Strukturen innerhalb 
der Frauenorganisationen sind, könnte mitunter eine Erklärung für die hohe Teilnahme der Frauen an den Protesten sein. Die Möglichkeit der hohen Teilnahme und die Präsenz der Frauen an den Protesten bedingt ebenfalls eine sich verändernde Vorstellung von Männlichkeit.

\section{Fazit}

Rückblickend kennzeichnet die Widerstandsformen der Feministinnen die konstruktive Auseinandersetzung mit der Protestsprache, die Organisation der stehenden-Frau-Performances, die Slogans und dass sie neuen Diskussionsthemen in die Foren einbrachten. Diese Aktionsformen tangierten in erster Linie unmittelbar die sexistischen Alltagspraktiken der Protestierenden im Park. Wie im ersten Teil dargestellt, ist die alternative Realität mit Rekurs auf den Sprachdiskurs im Park in erster Linie eine Reaktion auf die diskursiven Entwicklungen von der Vorstellung über „die richtige Familie“. Der Juni-Aufstand in der Türkei kann als Aufschrei gegenüber einer 12 Jahre währenden Phase aufgefasst werden, die einen Paradigmenwechsel mittels einer Neukonstituierung der Gesetzgebung und Neuorganisation des Staatsapparates zur Folge hatte. Welche Position dabei den Frauen zugewiesen wird, zeichnet den Machtkampf um die in der Gesellschaft verankerten Wertvorstellungen aus. Der schleichende Wechsel von einem Wertesystem zum anderen erreichte mit dem Ausbruch der Proteste seinen Höhepunkt. Durch die Proteste wurde die politische und wirtschaftliche Machtverschiebung in der Türkei sichtbar. Die vorgenommene Analyse hat gezeigt, dass die Umformung der Frauenrechte in marktkonforme Gesetzesregelungen und die Zunahme der Frauenfeindlichkeit in der Öffentlichkeit als ein Grund für den Ausbruch der Proteste unter vielen aufgeführt werden kann. Die Analyse zweier paralleler Diskurse, einerseits des öffentlichen Diskurses und andererseits der Park-Realität auf der Grundlage der Interviews, zeigte auf, welche Ausgangsbedingungen vorhanden waren, weshalb ein öffentlicher Park sich zu einem Ort der differierenden politischen Meinungen transformieren konnte. Die herangezogenen Interviewpassagen zeigen, wie eine asymmetrische Entwicklung (öffentlicher Diskurs versus Parkdiskurs) sich an einem Ort durchkreuzte und die gegenseitige Einflussnahme zeitigte. Das Aufeinanderprallen der beiden Diskurse kann als Reaktion auf eine sich non-stop transformierende politische Maschinerie gelesen werden, worauf die repressive Gewaltanwendung gegenüber den Protestierenden der Gezi-ParkBesetzung folgte. Die Kritik der feministischen Aktivist_innen an den vermeintlichen idealen Zuständen im besetzten Park, lässt zu, gesellschaftlich unsichtbare Mechanismen der Unterdrückung kritisch zu betrachten. Der Artikel soll dazu beitragen, den von der AKP-Regierung über die letzten 12 Jahre eingeleiteten 
und immer offensichtlicher werdenden Paradigmenwechsel, mit der Einbindung von islamischen Werten (d.h. religiösen Werten) der AKP-Wählerschaft in ein neoliberal ausgerichtetes Wirtschaftssystem und einen repressiven Staatsapparat, weiter sichtbar zu machen. Die Position der Frauen als Mütter der Nation und die damit einhergehende Verdrängung aus dem Öffentlichen zieht eine klare Grenze zwischen dem politischen und dem privaten Bereich. Damit wird eine stärkere gesellschaftlich regulierte Kategorie vom Frau-sein im öffentlichen Diskurs etabliert und davon abweichende Konstruktionen des Weiblich-seins als Normbruch eingestuft und sanktioniert.

\section{Literatur}

3 Çocuk Talebi (2013): Erdoğan 3 Çocuk Talebini Yineledi. URL: http://www.cnnturk.com/2013/ turkiye/01/02/erdogan.3.cocuk.talebini.yineledi/690965.0/, Zugriff: 12.10.2014.

Al-Rebholz, Anil (2007): Feminist Production of Knowledge and Redefinition of Politics in Turkey. In: Gerhard, Ute (Hg.): Wissenschaf $(f) t$ Geschlecht. Frankfurter feministische Texte, Frankfurt/M: 217-235.

- (2011): Das Ringen um die Zivilgesellschaft in der Türkei. Intellektuelle Diskurse, oppositionelle Gruppen und Soziale Bewegungen seit 1980, Bielefeld.

Ataç, Ilker (2009): Die 'Konservativ-Liberale' Politik Der AKP in Der Türkei im historischen Zusammenhang. In: Grundrisse. URL: linksnet.de/de/artikel/24835. Zugriff: 24.09.2014.

Babacan, Erol (2014): 'Shoppen, Beten, Kinderkriegen'-Aufstandinder Türkei. In: Infobrief Türkei. URL: infobrief-tuerkei.blogspot.de/2013/08/shoppen-beten-kinderkriegen-aufstand-in_6057. html, Zugriff: 15.08.2014.

Bakanlar Kurulu (2011): 8 Bakanlık gitti/6 Bakanlık Geldi. URL: ntvmsnbc.com/id/25221175/, Zugriff: 12.10.2014.

Eğitim (2012): Kızlar okuyunca erkekler evlenecek kız bulamıyor. URL: ohaber.com/-kizlarokuyunca-erkekler-evlenecek-kiz-bulamiyor--h-109179.html, Zugriff: 16.12.2014.

Eşit olamaz (2010): Kadın ve Erkek eşit olamaz, URL: haber.sol.org.tr/devlet-ve-siyaset/erdoganla-acilim-buraya-kadar-kadin-ve-erkek-esit-olamaz-haberi-31112, Zugriff: 17.12.2014.

Ev işi (2009): 'O Kafa' bakan olursa.URL: radikal.com.tr/politika/o_kafa_bakan_olursa-925986, Zugriff: 12.10.2014.

Faizsiz Çeyiz (2013): Erken evlenene Faizsiz Çeyiz, URL: alosgk.com/2013/07/erken-evlenenefaizsiz-ceyiz-kredisi-zaman-gazete, Zugriff: 16.12.2014.

Fıtrat Değil Anayasa (2014): Fıtrat değil Anayasa: Kadınlarve Erkekler Eşit Haklara Sahiptir. URL: cnnturk.com/haber/turkiye/fitrat-degil-anayasa-kadinlar-ve-erkekler-esit-haklara-sahiptir, Zugriff: 27.11.2014.

- (2014): Fıtrat değil Anayasa: Kadınlar ve erkekler eşit haklara sahiptir. URL: bianet.org/bianet/kadin/160287-fitrat-degil-anayasa-kadinlar-ve-erkekler-esit-haklara-sahiptir, Zugriff: 12.10.2014.

Günel, Sakine (2013): Dünden bugüne kürtaj hakkı! In: Feminist Politika, No. 19: 26-27.

HSYK (2011): „Tecavüzcüsüyle evlensin“ denmedi. URL: ntvmsnbc.com/id/25251420/,Zugriff: 12.10.2014.

Kadın Evlensin (2011): Kadin Tecavüzcüsüyle Evlensin. URL: ntvmsnbc.com/id/25251279, Zugriff: 14.10.2014.

Kadın Hukuku (2003): Adım adım kadın-erkek Eşitliği. URL: turkhukuksitesi.com/showthread. php?t=2740, Zugriff: 4.1.2015. 
Kadın işleri (2009): Kriz zamanı kıymete binen Kadın işleri. URL: bianet.org/bianet/ emek/113903-kriz-zamani-kiymete-binen-kadin-isleri, Zugriff: 12.10.2014.

Kadın istihdamı (2009): Kadın istihdamı. URL: bianet.org/bianet/siyaset/113252-erbatur-bakansimsek-i-kadin-istihdami-konusunda-elestirdi, Zugriff: 12.10.2014.

Kadın Örgütleri (2011): Kadın Örgütleri öfkeli. URL: bianet.org/bianet/kadin/130585-kadinbakanligi-kaldirildi-kadin-orgutleri-ofkeli, Zugriff: 16.12.2014.

Karakaş, Cemal (2013): Das Demokratieverständnis Der Türkischen Gerechtigkeits- und Entwicklungspartei (AKP). In: Rill, Bernd (Hg.): Türkische Innenpolitik. Abschied vom Kemalismus, München: 21-29.

Kendini öldürsün (2012): Melih Gökçek: Anası kendini öldürsün. URL: radikal.com.tr/turkiye/ melih_gokcek_anasi_kendini_oldursun-1089899, Zugriff: 16.12.2014.

Kürtaj açıklaması (2012): Bakan Fatma Şahin: Kürtaj açıklaması. URL: milliyet.com.tr/bakanfatma-sahin-den-kurtaj-aciklamasi/siyaset/siyasetdetay/30.05.2012/1547015/default.htm, Zugriff: 16.12.2014.

Kürtaj Uluderedir (2012): Erdoğan: Her kürtaj bir uluderedir. URL: cnnturk.com/2012/turkiye/05/26/erdogan.her.kurtaj.bir.uluderedir/662524.0/, Zugriff: 16.12.2014.

Sirman, Nükhet (2008): Die Familisierung der Staatsbürgerschaft in der Türkei, in Perspektiven auf die Türkei: ökonomische und gesellschaftliche (Dis)Kontinuitäten im Kontext der Europäisierung. Münster: 296-321.

Taksim Dayanı̧̧ması (2014). URL: taksimdayanisma.org/bilesenler?lang=en, Zugriff: 15.12.2014.

Tecavüz Bebeği (2012): Bakan Akdağ: Tecavüz Bebeğine devlet bakar. URL: radikal.com.tr/ turkiye/bakan_akdag_tecavuz_bebegine_devlet_bakar-1089651, Zugriff: 16.12.2014.

Tolunay, Özlem Ilyas (2014): Women in Erdoğans Turkey. In: New Politics. URL: newpol.org/ content/women-erdoğan's-turkey, Zugriff: 15.12.2014.

Umul, Fatma (2013): Nimm deine Hände von meinem Körper! Erdoğans reaktionäre Familienpolitik tabuisiert Gewalt gegen Frauen, in: ak - analyse \& kritik, Nr. 585.

- (2014): Mit dem Schuhkarton auf sie Demo. Erdoğan gewinnt die Kommunalwahlen trotz Korruption und Gezi-Protesten, in: ak - analyse \& kritik, Nr. 593. URL: akweb.de/ak_s/ ak593/17.htm, Zugriff: 12.10.2014.

Zina Yasası (2004): Erdoğan: Zina Yasası Eşitsizliği Giderecek. URL: cnnturk.com/2004/turkiye/09/04/erdogan.zina.yasasi.esitsizligi.giderecek/33272.0/, Zugriff: 16.12.2014. 